\title{
Synergism of Oxacillin and Gentamicin Against Enterococci
}

\author{
ROBERT L. MARIER,* NANCY JOYCE, AND VINCENT T. ANDRIOLE \\ Department of Internal Medicine, Yale University School of Medicine, New Haven, Connecticut 06510
}

Received for publication 16 July 1975

\begin{abstract}
Strains of enterococci isolated from 34 patients were studied for susceptibility to oxacillin and gentamicin alone and in combination. The minimal bactericidal concentrations of oxacillin and gentamicin for these strains ranged from 16 to $250 \mu \mathrm{g} / \mathrm{ml}$ (median $32 \mu \mathrm{g} / \mathrm{ml}$ ) for oxacillin and 12 to $48 \mu \mathrm{g} / \mathrm{ml}$ (median $24 \mu \mathrm{g} / \mathrm{ml}$ ) for gentamicin. The minimal bactericidal concentration of oxacillin for $50 \%$ of strains in the presence of $12,6,3$, and $1.5 \mu \mathrm{g}$ of gentamicin per ml, respectively, was $2,8,16$, and $32 \mu \mathrm{g} / \mathrm{ml}$. The combination of oxacillin and gentamicin at clinically attainable serum levels was synergistically bactericidal against $27 / 34(80 \%)$ of these strains using strict criteria. This report reconfirms the original observation that antibiotics which affect the synthesis of bacterial cell walls combine synergistically with aminoglycosides against enterococci. This is so even though enterococci are far more resistant to oxacillin than to penicillin or ampicillin. In addition, this report suggests that the combination of oxacillin and gentamicin administered in the usual dosages includes enterococci in its bactericidal spectrum.
\end{abstract}

There have been a number of reports in recent years on the in vitro effectiveness of penicillin and its derivatives combined with aminoglycosides against enterococci. It has been shown that a synergistic effect is achieved when the enterococcal strain is relatively susceptible to the aminoglycoside in the combination (10). Since some strains are highly resistant to streptomycin and kanamycin, combinations of these aminoglycosides with penicillin are not synergistic against all enterococci $(12,16)$. No strains have been reported to be highly resistant to gentamicin. This information has been useful in the selection of antibiotics for the treatment of enterococcal endocarditis wherein cure rates may correlate with the use of synergistic combinations (15).

Other combinations of antibiotics which effect bacterial cell wall synthesis, such as cycloserine, bacitracin, vancomycin, cephalothin, methicillin, oxacillin, and ampicillin, have been shown to have a synergistic effect in combination with streptomycin against many strains of enterococci $(9,13)$. Vancomycin and gentamicin has been shown to be effective against more strains of enterococci than vancomycin and streptomycin (14). Carbenicillin and gentamicin has been shown to be synergistic against enterococci as well (7). Since the combination of oxacillin and gentamicin might be used as emperic therapy or in mixed infections, its activity against enterococci is of interest. This combination was recently reported to be synergistic against 3/14 strains of enterococci using differ- ent criteria of synergy (time killing curves) than those used in this study (checkerboard technique) (4). In that study, nafcillin and gentamicin were more effective than oxacillin and gentamicin against enterococci.

\section{MATERIALS AND METHODS}

Enterococci. Thirty-four strains of enterococci isolated from a variety of sites in different patients were obtained from the clinical microbiology laboratory at the Yale-New Haven Hospital. All strains were identified by the usual growth criteria (2). They were gram-positive cocci producing nonhemolytic or $\gamma$-hemolytic colonies on sheep blood agar. They grew in both $6.5 \% \mathrm{NaCl}$ broth and bile esculin agar and hydrolized sodium hippurate. Serologic testing was not done.

Antibiotics. Sodium oxacillin standard powder was supplied by Bristol Laboratories, Syracuse, N.Y. Gentamicin sulfate laboratory standard was supplied by Schering Corporation, Bloomfield, N.J.

Synergy studies. A checkerboard technique was employed as previously reported (1). The final concentrations of oxacillin ranged from 500 to 0.25 $\mu \mathrm{g} / \mathrm{ml}$, and gentamicin ranged from 12 to $0.19 \mu \mathrm{g} / \mathrm{ml}$. The minimal inhibitory concentration (MIC) was defined as the lowest concentration of one drug that prevented gross turbidity upon visual inspection after overnight incubation at $37 \mathrm{C}$. From the clear wells, approximately $10 \mu \mathrm{l}$ of broth was removed and subcultured on sheep blood agar. The minimal bactericidal concentration (MBC) was designated as the lowest concentration of one drug that prevented growth on subculture. All studies were conducted in triplicate and repeated if necessary until a majority of assays were in agreement for any one strain. A reduction of the MIC or MBC of both antibiotics by 
fourfold was defined as synergy, whereas a similar reduction of one antibiotic and a twofold reduction of the MIC or MBC of the other antibiotic was defined as partial synergy. Indifference was defined as a twofold reduction in the MIC or MBC of only one antibiotic and antagonism as no reduction or an increase in the MIC or MBC of either antibiotic. When the MIC or MBC of gentamicin exceeded 12 $\mu \mathrm{g} / \mathrm{ml}$ (the highest concentration on the microtiter plate), quadruplicate determinations of the MIC and MBC were made using gentamicin concentrations ranging from 0.10 to $192 \mu \mathrm{g} / \mathrm{ml}$.

\section{RESULTS}

MIC and MBC of oxacillin and gentamicin. The cumulative percentage of strains killed hibited (MIC) or killed (MBC) by oxacillin and gentamicin used alone is summarized in Fig. 1. The MBCs of oxacillin and gentamicin for these strains ranged from 16 to $250 \mu \mathrm{g} / \mathrm{ml}$ (median 32 $\mu \mathrm{g} / \mathrm{ml}$ ) for oxacillin and 12 to $48 \mu \mathrm{g} / \mathrm{ml}$ (median $24 \mu \mathrm{g} / \mathrm{ml}$ ) for gentamicin.

MBC of oxacillin in presence of gentamicin. The cumulative pecentage of strains killed by oxacillin in the presence of varying amounts of gentamicin is summarized in Fig. 2. The MBC of oxacillin for $50 \%$ of strains in the presence of $12,6,3$, and $1.5 \mu \mathrm{g}$ of gentamicin per $\mathrm{ml}$, respectively, was $2,8,16$, and $32 \mu \mathrm{g} / \mathrm{ml}$. At these same concentrations of gentamicin, respectively, $100 \%$ of strains were killed by 16,32 , and $64 \mu \mathrm{g} / \mathrm{ml}$.

Synergism. The bacteriostatic and bactericidal effects of oxacillin and gentamicin combinations are summarized in Fig. 3. In this figure, the concentrations of the antibiotic are expressed as a percentage of the MBC of each drug alone. For example, a fourfold reduction of the $\mathrm{MBC}$ is equal to $25 \%$ of the MBC. The combination was synergistically bactericidal

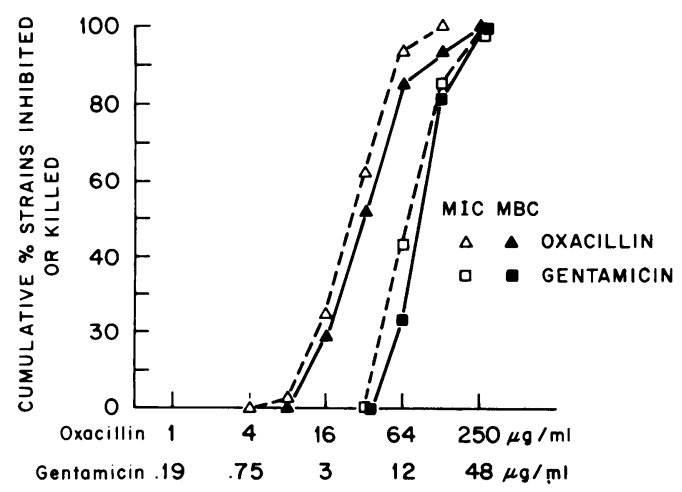

FIG. 1. Cumulative percentage of strains of enterococci inhibited (MIC) or killed $(M B C)$ by varying amounts of oxacillin and gentamicin used alone.

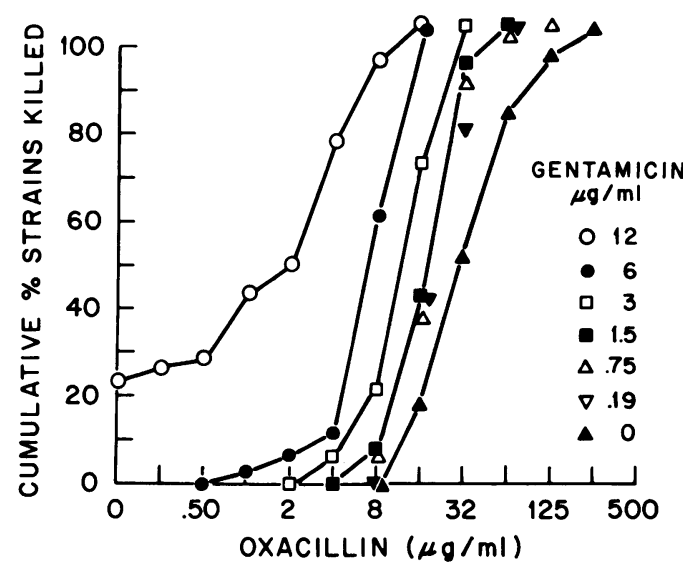

Fig. 2. Cumulative percentage of strains of enterococci killed by oxacillin in the presence of varying amounts of gentamicin.

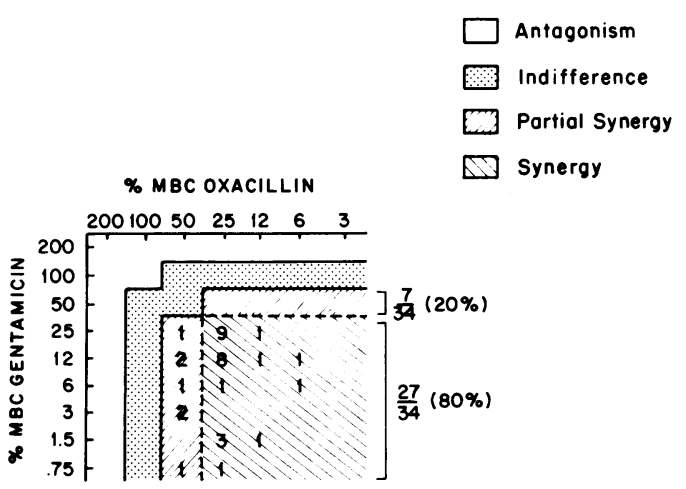

Fig. 3. Number of enterococcal strains killed by various combinations of oxacillin and gentamicin.

against $27 / 34(80 \%)$ of strains tested using the more strict criteria and against 7/34 (20\%) using the less strict criteria (see above). In four instances, the degree of synergism was striking with MBCs of each antibiotic reduced by eightfold (12\% MBC) or more. MIC data were comparable. There is no correlation between the degree of synergy and the MIC or MBC of either antibiotic. An example of an isobologram demonstrating the synergism of this combination is shown in Fig. 4.

\section{DISCUSSION}

The selection of any combination of antibiotics to be used as emperic therapy depends on the probability that the likely organism(s) causing the infection is inhibited or killed by the combination. Hence, we analyzed the susceptibility of enterococci to various combinations of oxacillin and gentamicin in terms of frequency distribution. When used alone, oxacillin or gen- 


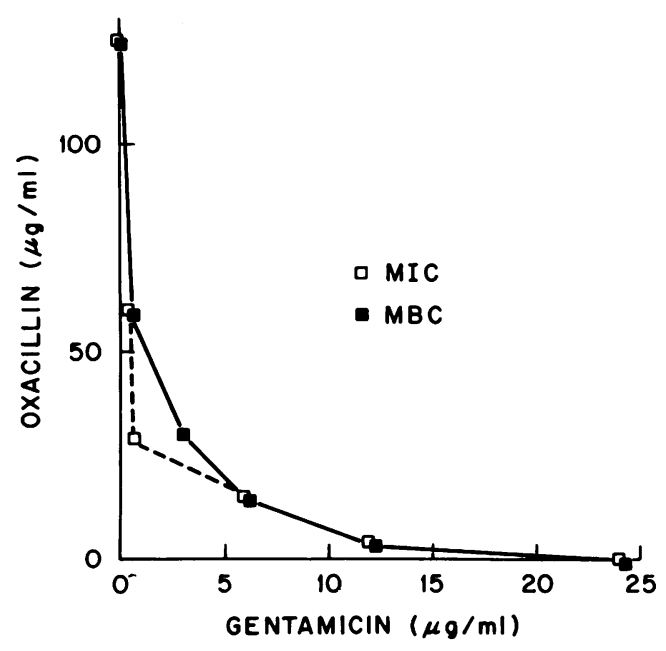

Fig. 4. Effect of oxacillin and gentamicin on one enterococcal strain.

tamicin would have killed less than $50 \%$ of strains at concentrations of 32 and $12 \mu \mathrm{g} / \mathrm{ml}$, respectively. These concentrations are outside those usually attained in the serum $(3,6)$. In combination, however, oxacillin and gentamicin killed $100 \%$ of strains at half these concentrations. Smaller amounts of either antibiotic were often effective in combination. Oxacillin and gentamicin, in the usual doses, includes enterococci in its bactericidal spectrum.

A second type of analysis was done for more theoretical purposes. In vitro antibacterial synergism may be defined by killing curves or isobolograms $(5,11)$. There are no clear advantages of either method. When the MBC is used to construct the isobologram the results are comparable (1). The later method was used in this study. Oxacillin and gentamicin were shown to have a synergistic bactericidal effect according to the more strict criteria against $27 / 34(80 \%)$ of strains of enterococci studied. This observation reconfirms the original report that aminoglycosides combined with antibiotics which interfere with cell wall synthesis affect enterococci synergistically (9). This is so even though enterococci are clearly more resistant to oxacillin than to penicillin or ampicillin (me- dian MICs, 50, 3.1, and $1.6 \mu \mathrm{g} / \mathrm{ml}$, respectively; 8).

\section{LITERATURE CITED}

1. Andriole, V. T. 1971. Synergy of cargenicillin and gentamicin in experimental infection with pseudomonas. J. Infect. Dis. 124S:46-55.

2. Facklam, R. R. 1973. Comparison of several laboratory media for presumptive identification of enterococci and group D streptococci. Appl. Microbiol. 26:138145.

3. Gingell, J. C., G. D. Chisholm, J. S. Calnan, and P. M. Waterworth. 1969. The dose, distribution, and excretion of gentamicin with special reference to renal failure. J. Infect. Dis. 119:396-401.

4. Glew, R. H., R. C. Moellering, and C. Wennersten. 1975. Comparative synergistic activity of nafcillin, oxacillin, and methicillin in combination with gentamicin against enterococci. Antimicrob. Agents Chemother. 7:828-832.

5. Jawetz, E. 1968. Combined antibiotic action: some definitions and correlations between laboratory and clinical results, p. 203-209. Antimicrob. Agents Chemother. 1967.

6. Klein, J. O., L. D. Sabath, and M. Finland. 1963. Laboratory studies on oxacillin. Am. J. Med. Sci. 245:399411.

7. Libke, R. D., C. Regamey, J. T. Clarke, and W. M. Kirby. 1973. Synergism of carbenicillin and gentamicin against enterococci. Antimicrob. Agents Chemother. 4:564-568.

8. March, W., and J. Klein. 1970. The isoxazolyl penicillins: oxacillin, cloxicillin, and dicloxacillin. Med. Clin. North Am. 54:1127-1143.

9. Moellering, R. C., C. Wennersten, and A. N. Weinberg. 1971. Studies on antibiotic synergism against enterococci. J. Lab. Clin. Med. 77:821-828.

10. Moellering, R. C., C. Wennersten, and A. N. Weinberg. 1971. Synergy of penicillin and gentamicin against enterococci. J. Infect. Dis. 124S:207-209.

11. Sabath, L. D. 1968. Synergy of antibacterial substances by apparently known mechanisms, p. 210-217. Antimicrob. Agents Chemother. 1967.

12. Standiford, H. D., J. B. de Maine, and W. M. Kirby. 1970. Antibiotic synergism of enterococci. Arch. Intern. Med. 126:255-259.

13. Watanakunakorn, C. 1971. Penicillin combined with gentamicin or streptomycin: synergism against enterococci. J. Infect. Dis. 124:581-586.

14. Watanakunakorn, C., and C. Bakie. 1973. Synergism of vancomycin-gentamicin and vancomycin-streptomycin against enterococci. Antimicrob. Agents Chemother. 4:120-124.

15. Weinstein, A. J., and R. C. Moellering. 1973. Penicillin and gentamicin therapy for enterococcal infection. $J$. Am. Med. Assoc. 223:1030-1032.

16. Wilkowske, C. J., R. R. Facklam, J. A. Washington, and J. E. Geraci. 1972. Antibiotic synergism: enhanced susceptibility of group D streptococci to certain antibiotic combinations, p. 195-200. Antimicrob. Agents Chemother. 1971. 